\title{
Unsteady MHD flow of a micropolar nanofluid through a dilating channel in the presence of ohmic heating
}

\author{
R. V. S. R. K. Sastry $D^{1}$, Naresh Kumar $N^{1}$, Sivaraj R ${ }^{2}$, and Animasaun I.L. ${ }^{3}$ \\ ${ }^{1}$ SASTRA University \\ ${ }^{2}$ Vellore Institute of Technology \\ ${ }^{3}$ Federal University of Technology, Akure, Nigeria
}

June 6, 2020

\begin{abstract}
Heat transfer in a magnetohydrodynamic (MHD) micropolar fluid through a squeezing channel in the presence of viscous dissipation and Joule heating is studied. The analysis is carried out over different water based nanofluids consisting of alumina (Al2O3), titania ( $\mathrm{TiO} 2)$, and magnetite (Fe3O4) nanoparticles. A model with non-linear partial differential equations is converted to a set of non-linear ordinary differential equations by the aid of similarity transformations. The resulting equationsare then numerically solved by Runge-Kutta (RK) method with fourth order approximation followed by shooting method. A comprehensive investigation describing the influences of the physical parameters such as vortex viscosity parameter, ohmic heating parameter, Hartmann number, squeezing parameter and solid volume fraction parameter on the distributions of temperature, velocity and microrotationis are graphically showed. In the absence of squeezing and expanding, it is observed that both water and nanofluid experience same viscous drag. Further, an increase in volume fraction lowers the microrotation, and thereby causing an elevation in the temperature of titania-water nanofluid. This is in contrast to the behaviour of the other nanofluids.
\end{abstract}

\section{Hosted file}

Manuscript_final.doc available at https ://authorea.com/users/330657/articles/457452-unsteadymhd-flow-of-a-micropolar-nanofluid-through-a-dilating-channel-in-the-presence-of-ohmicheating

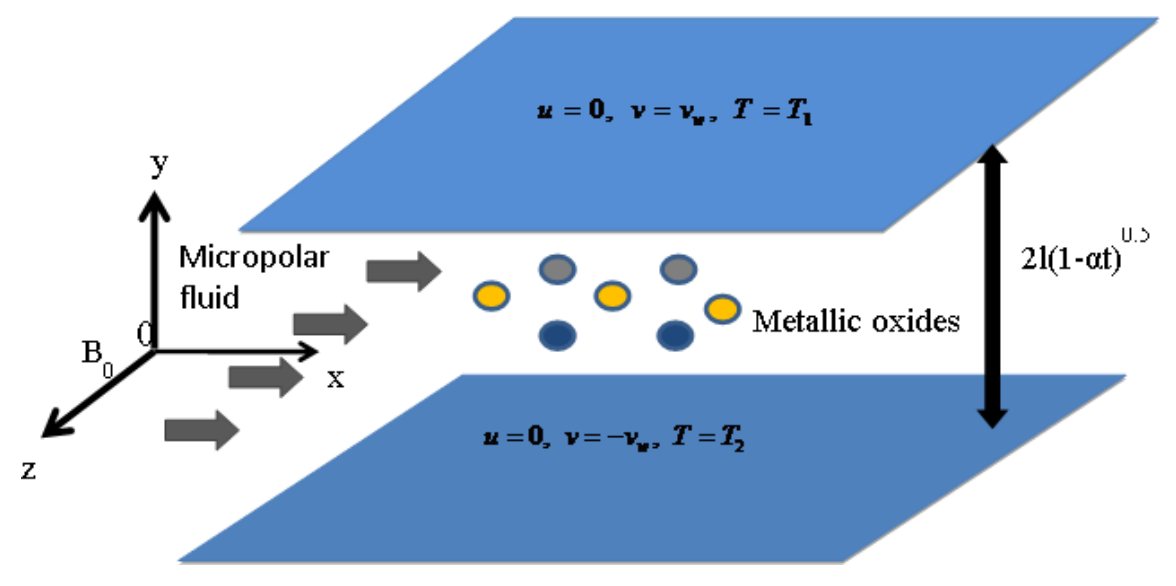



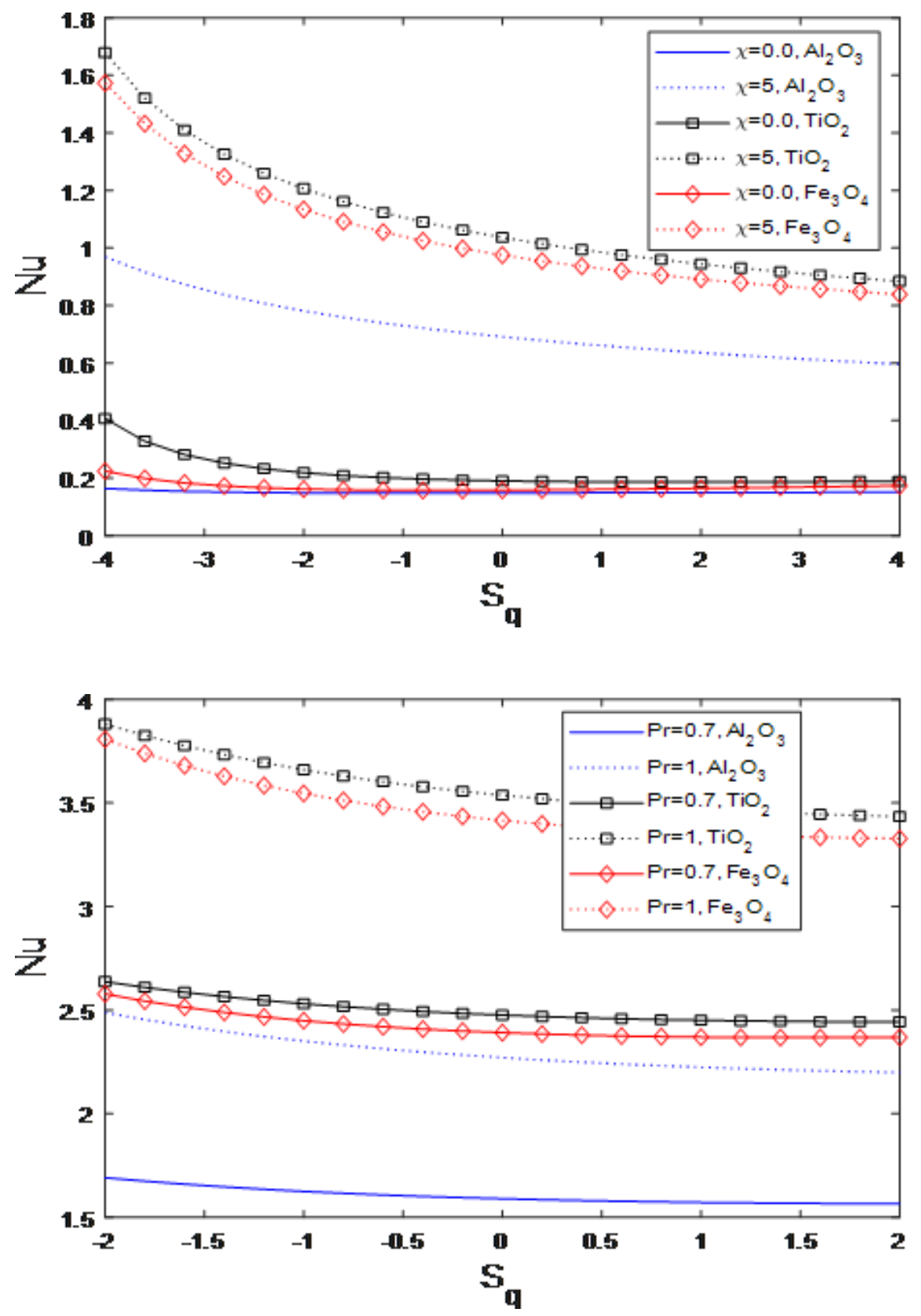

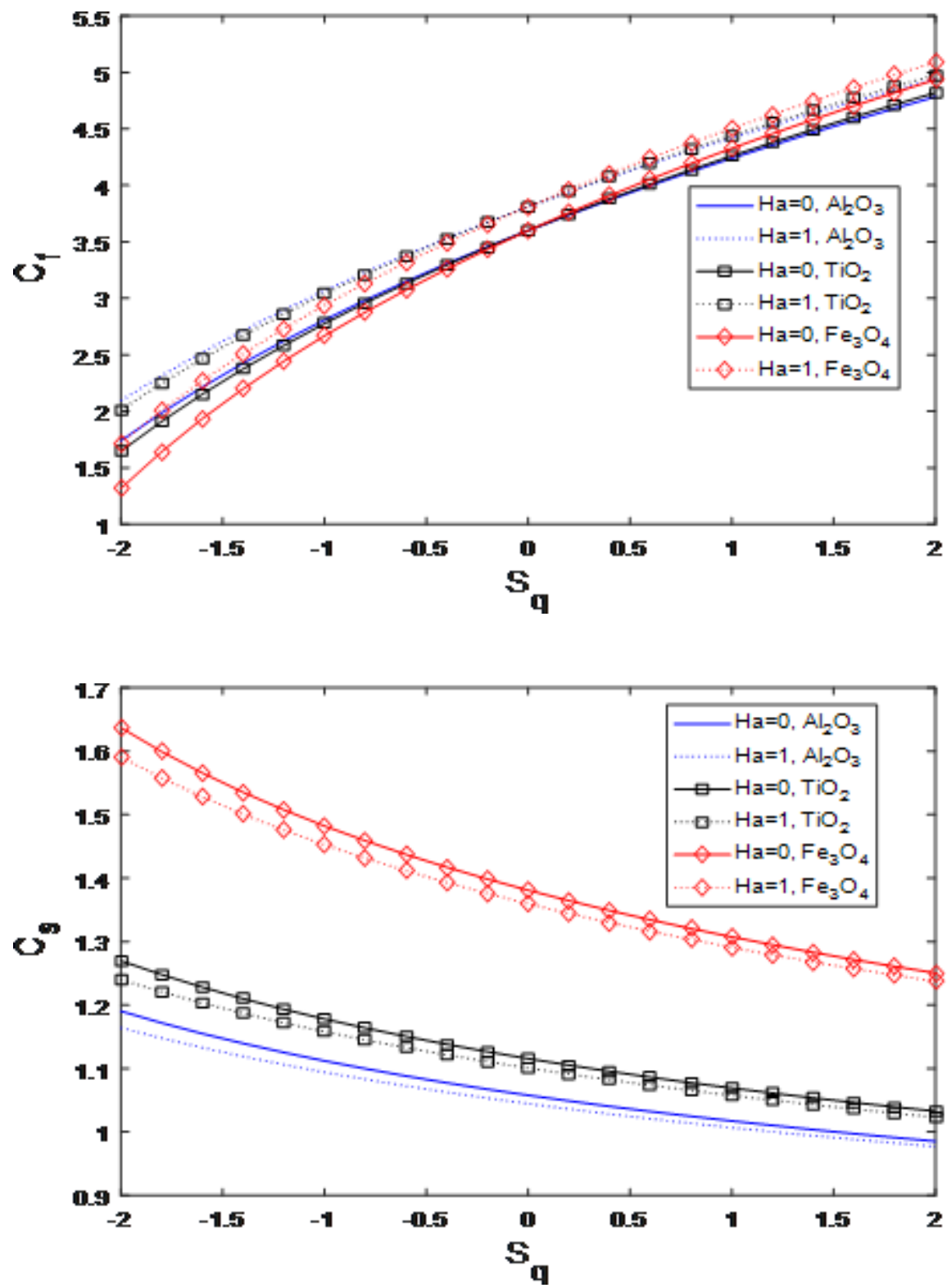

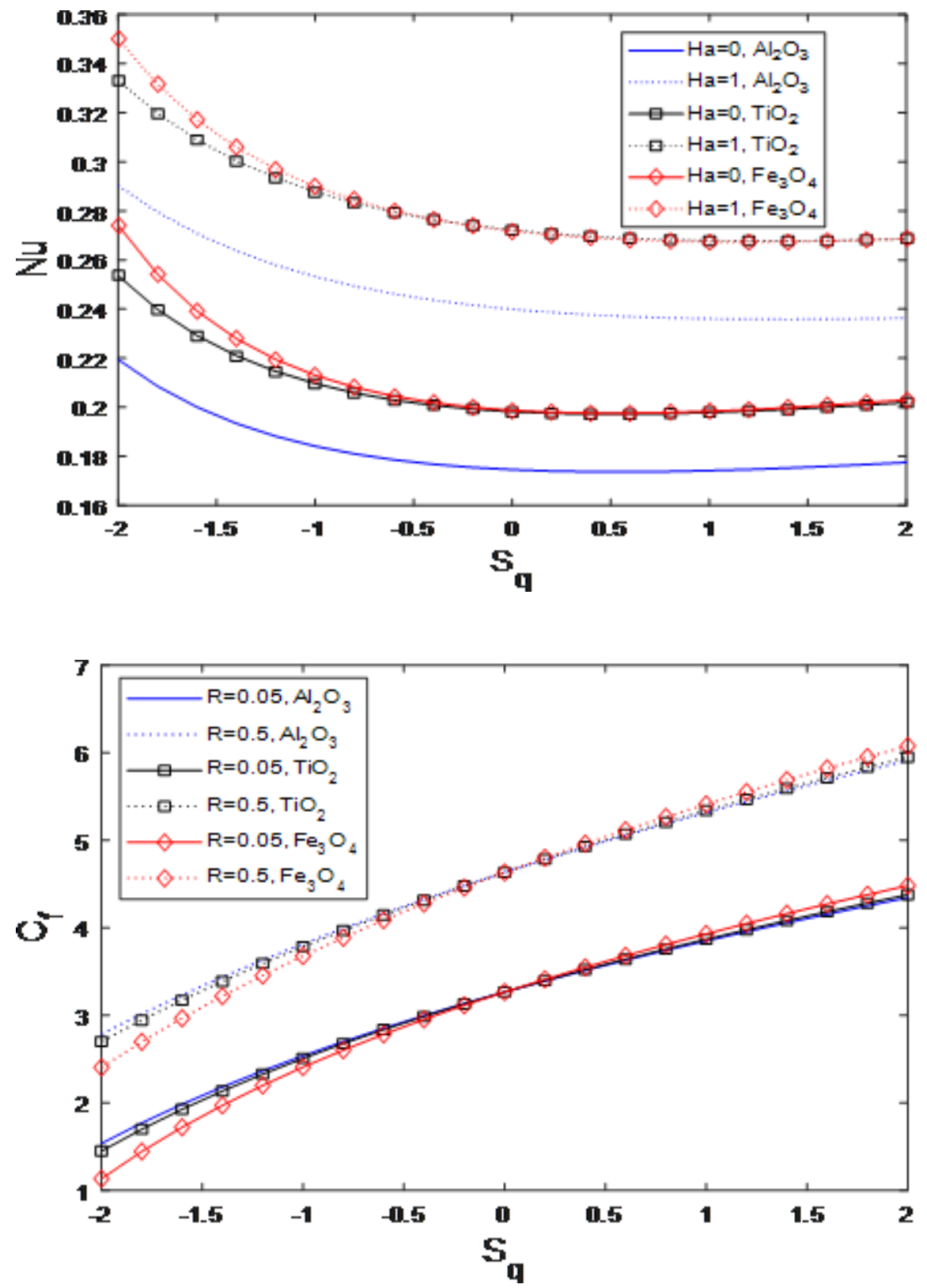

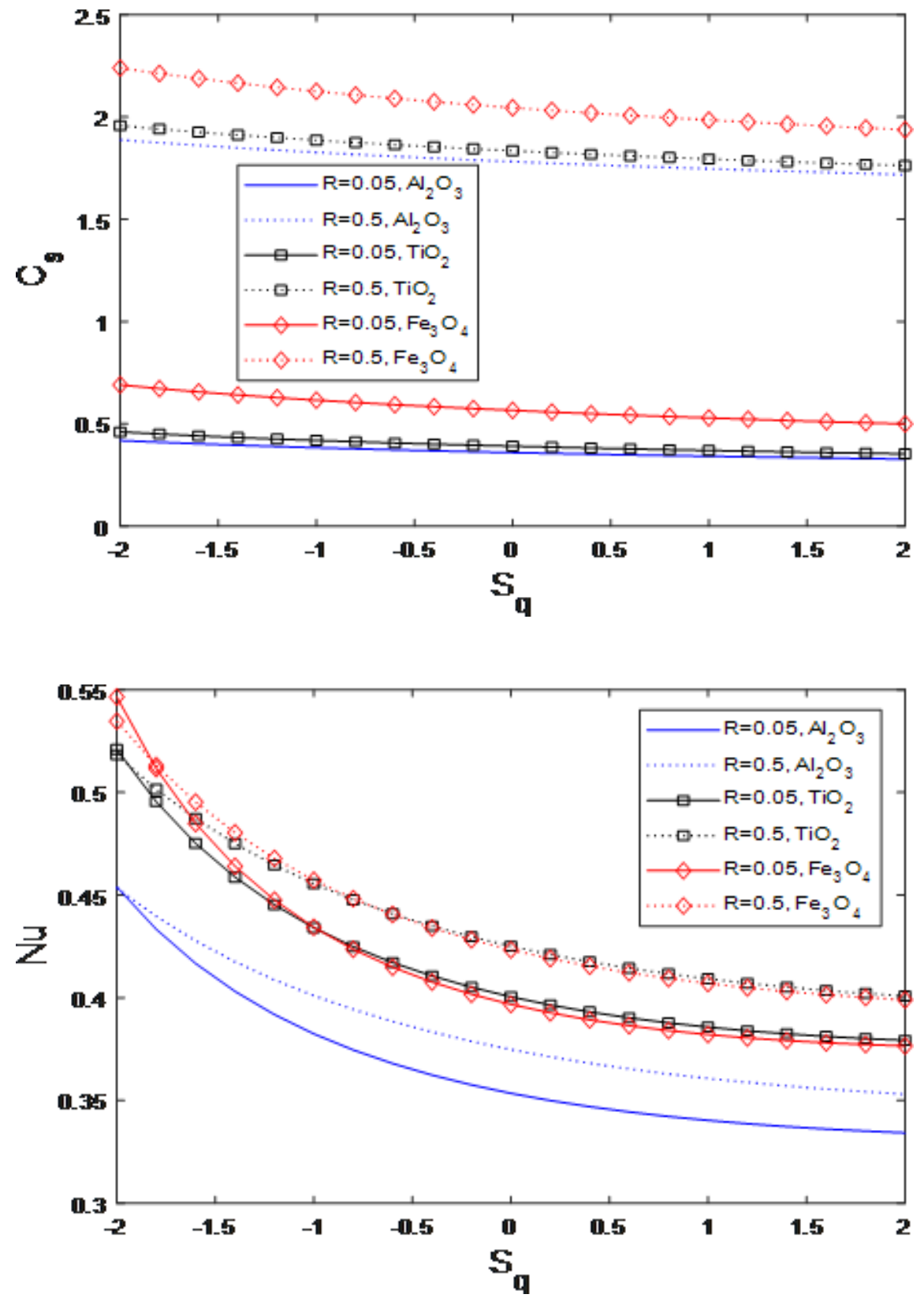

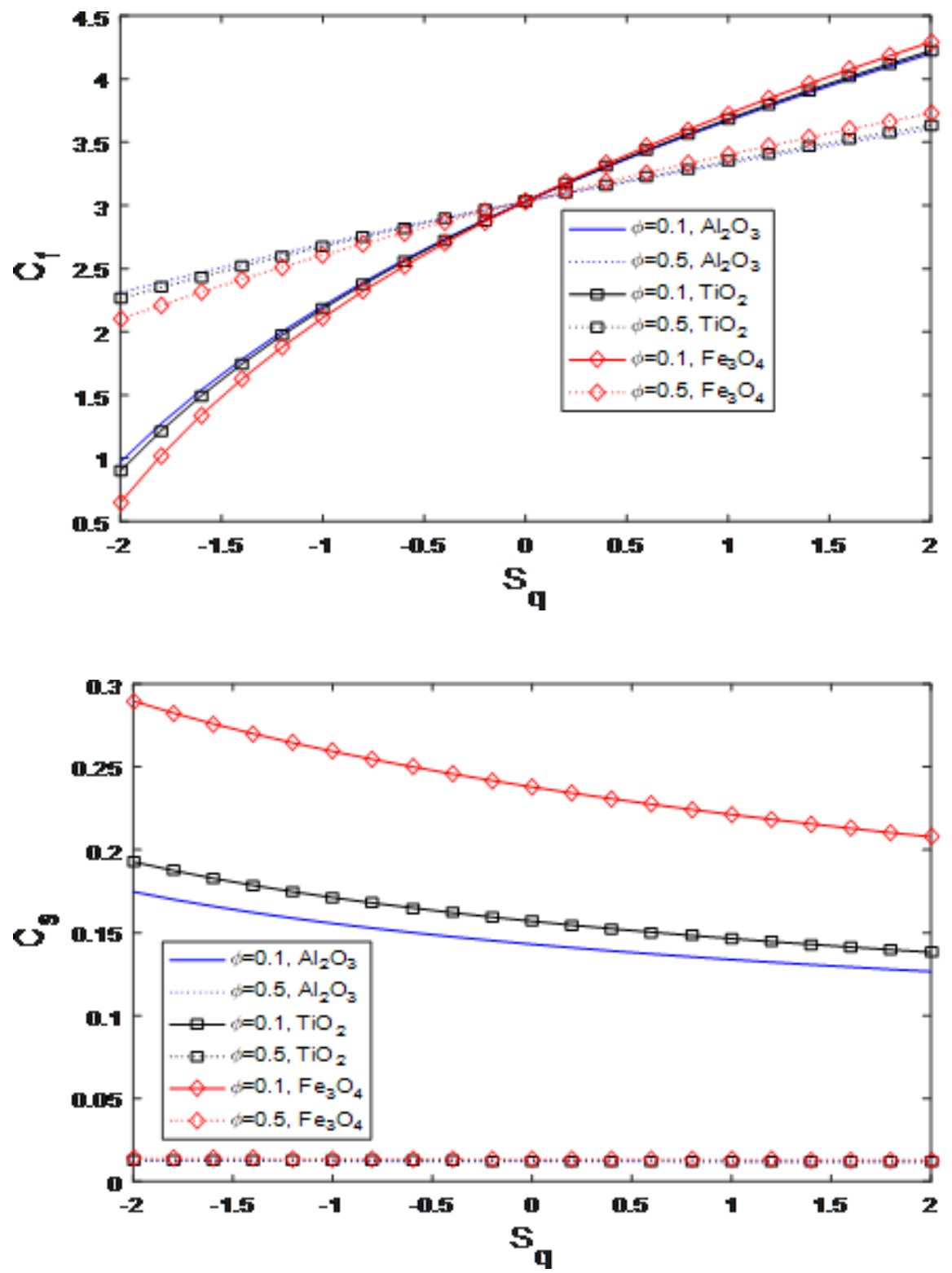

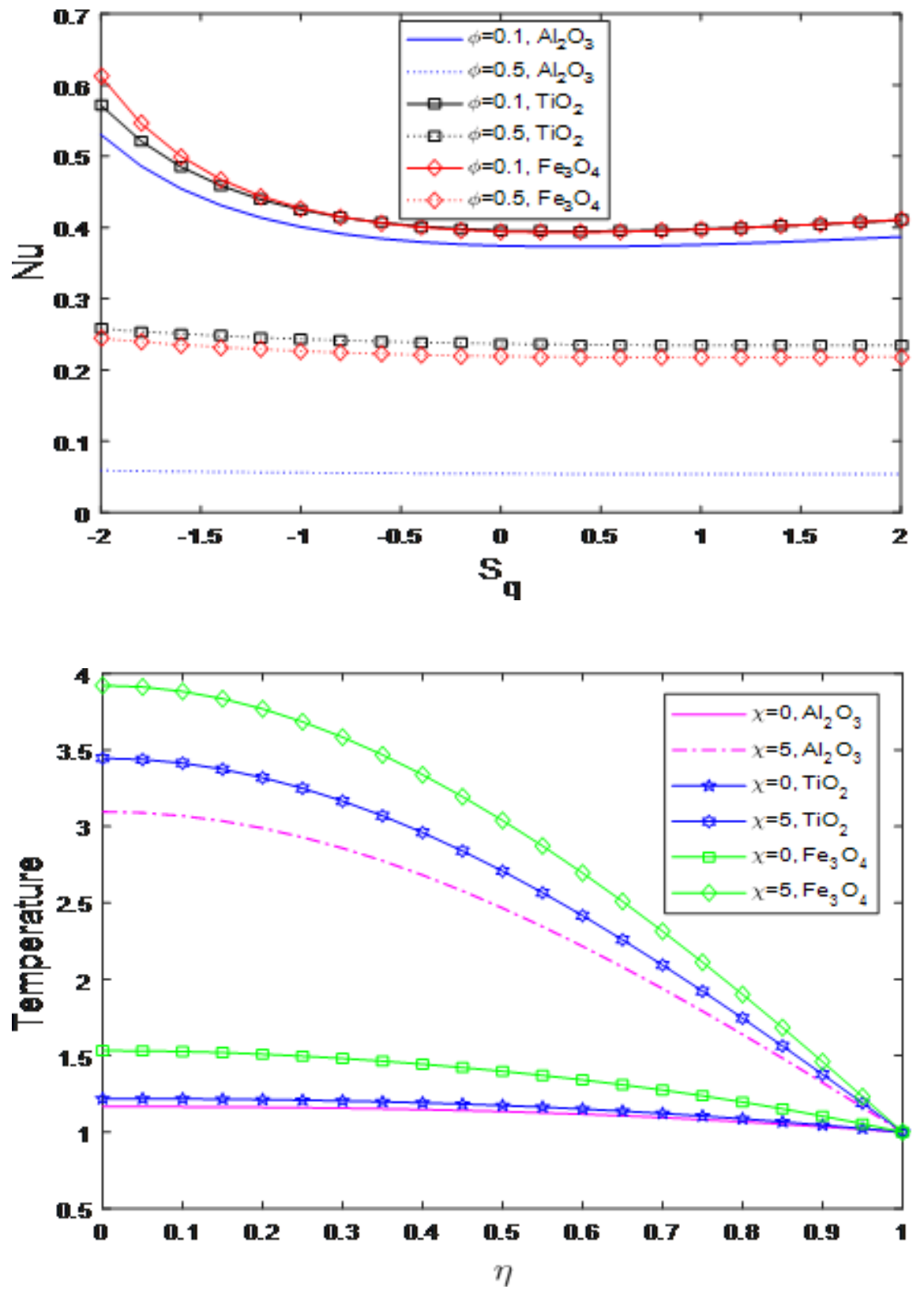

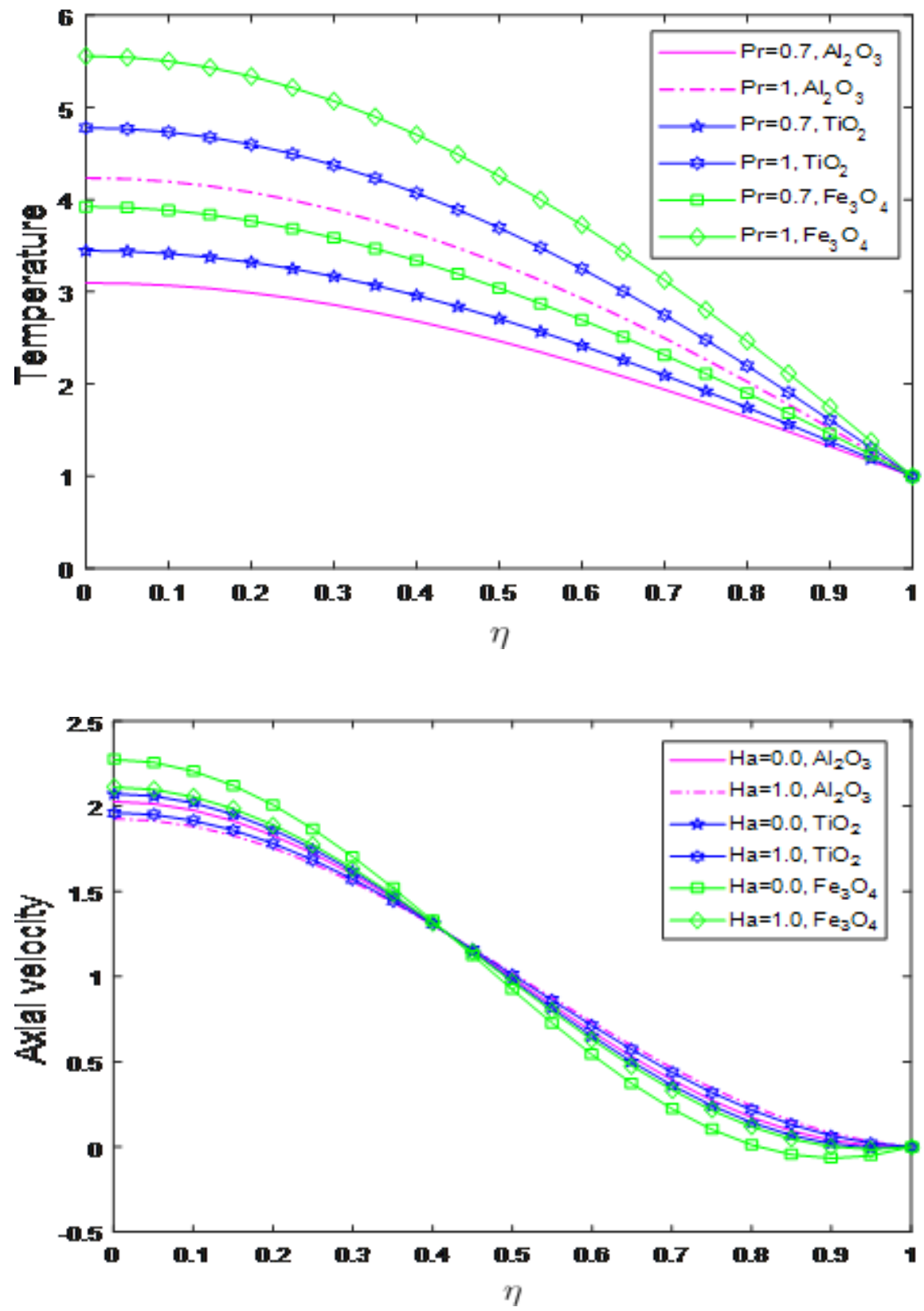

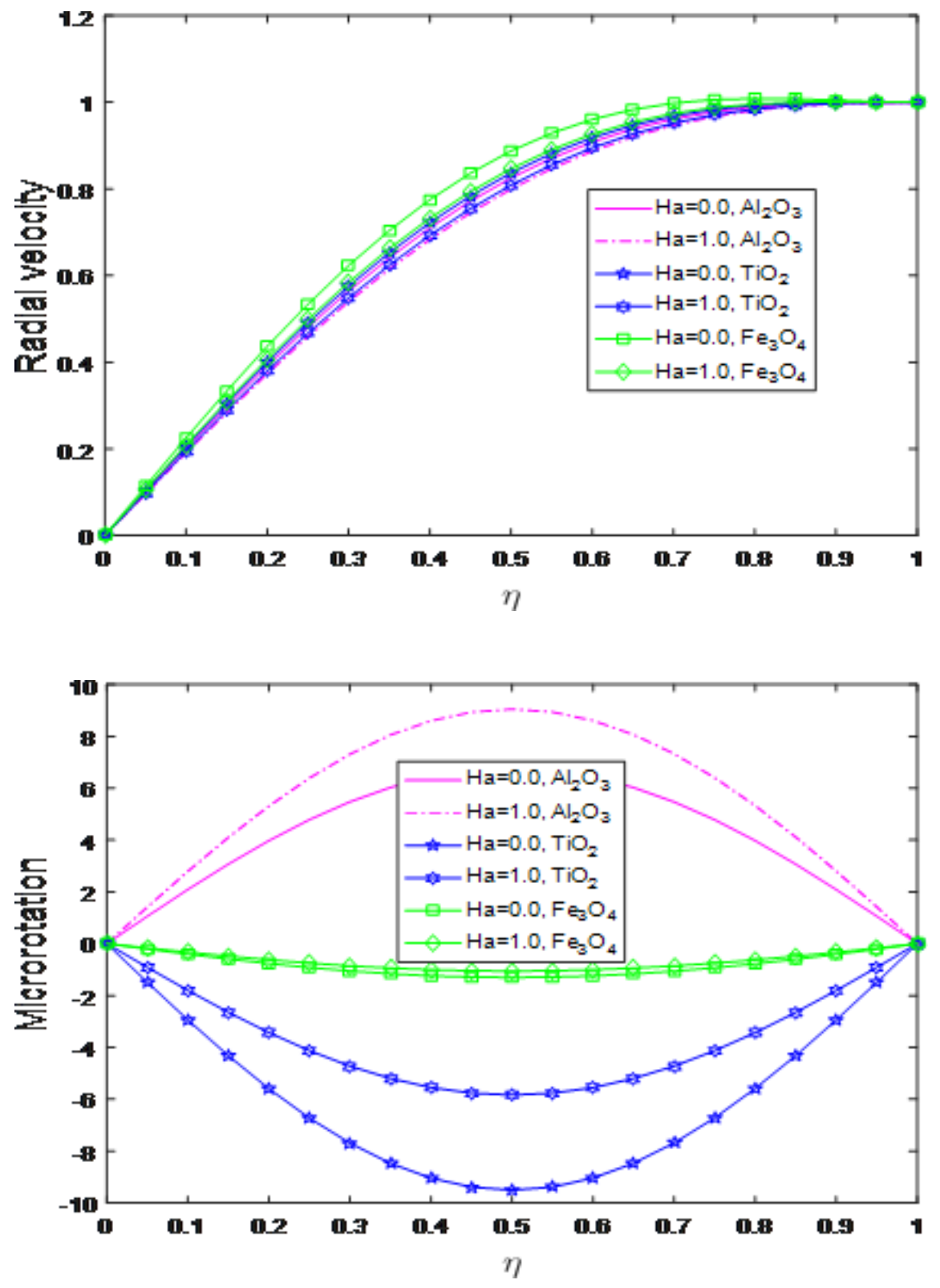

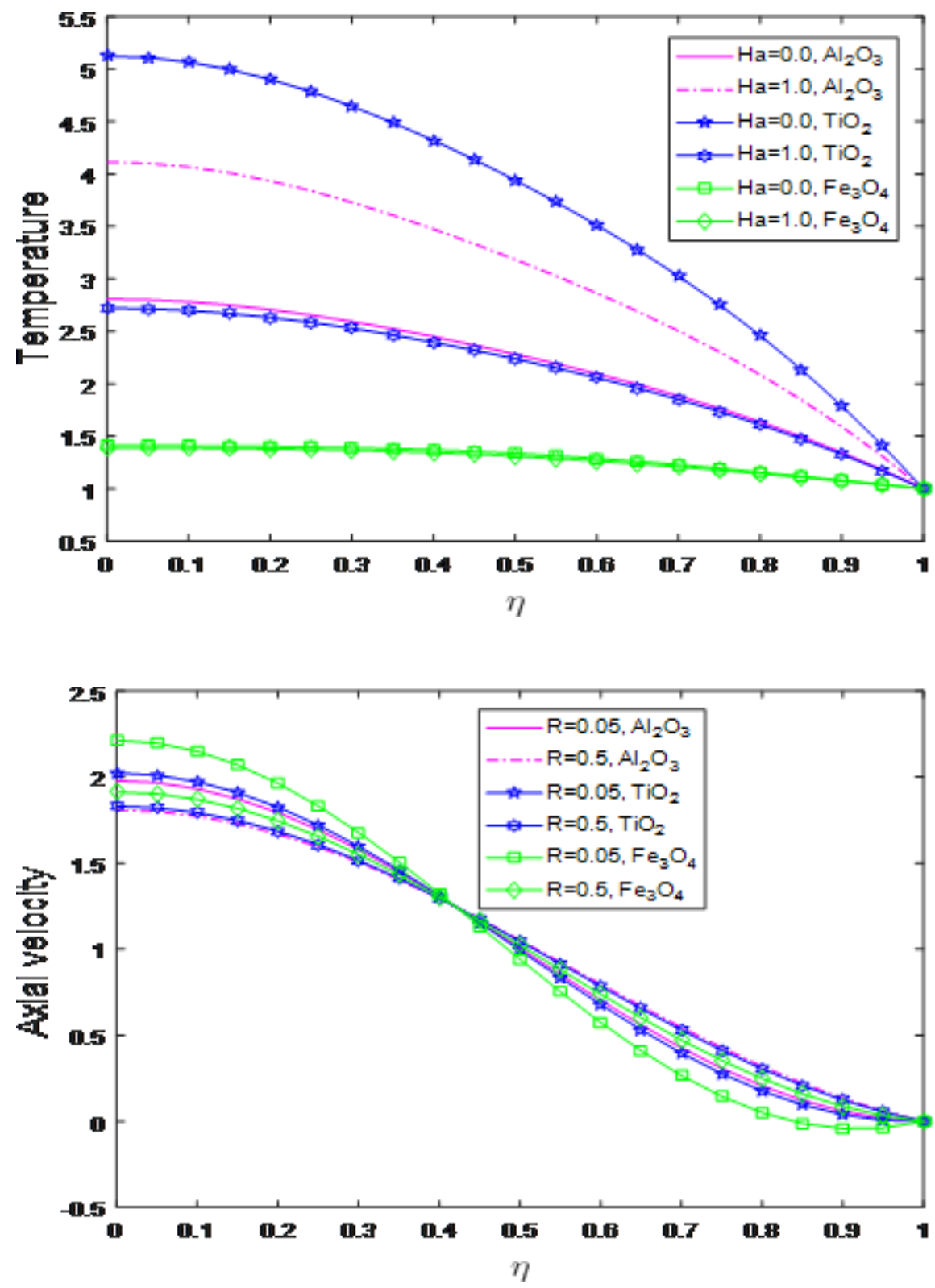

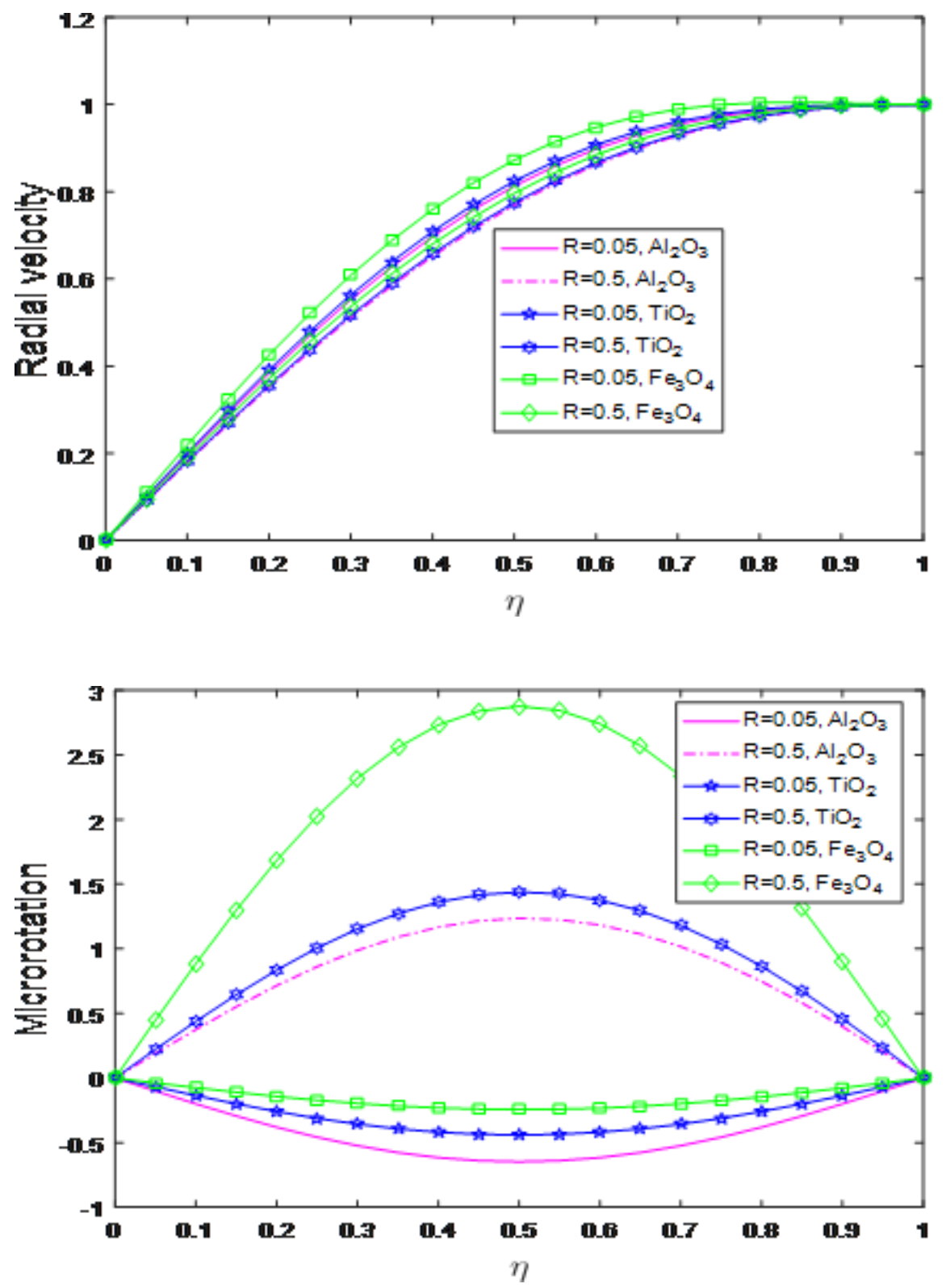

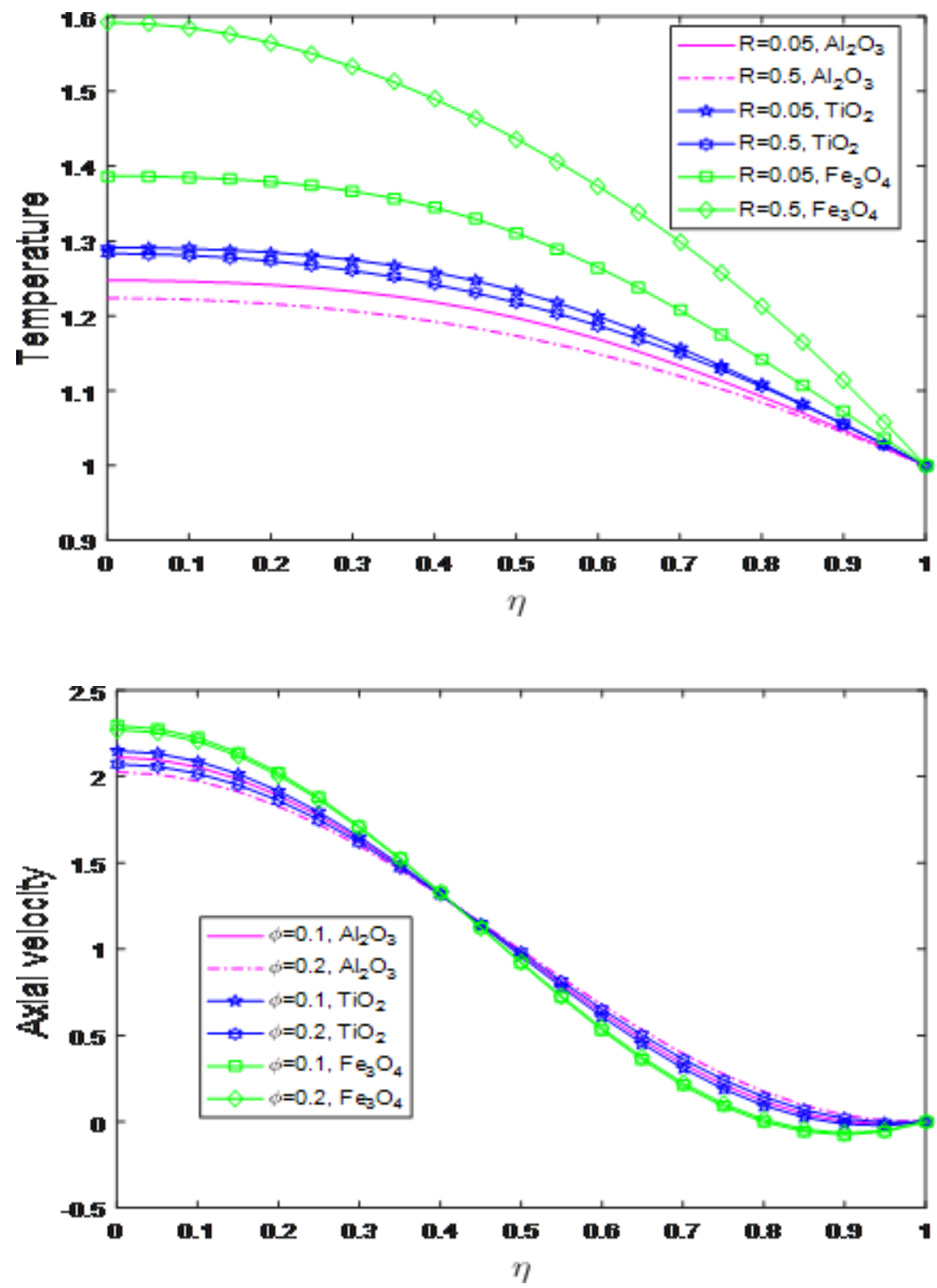

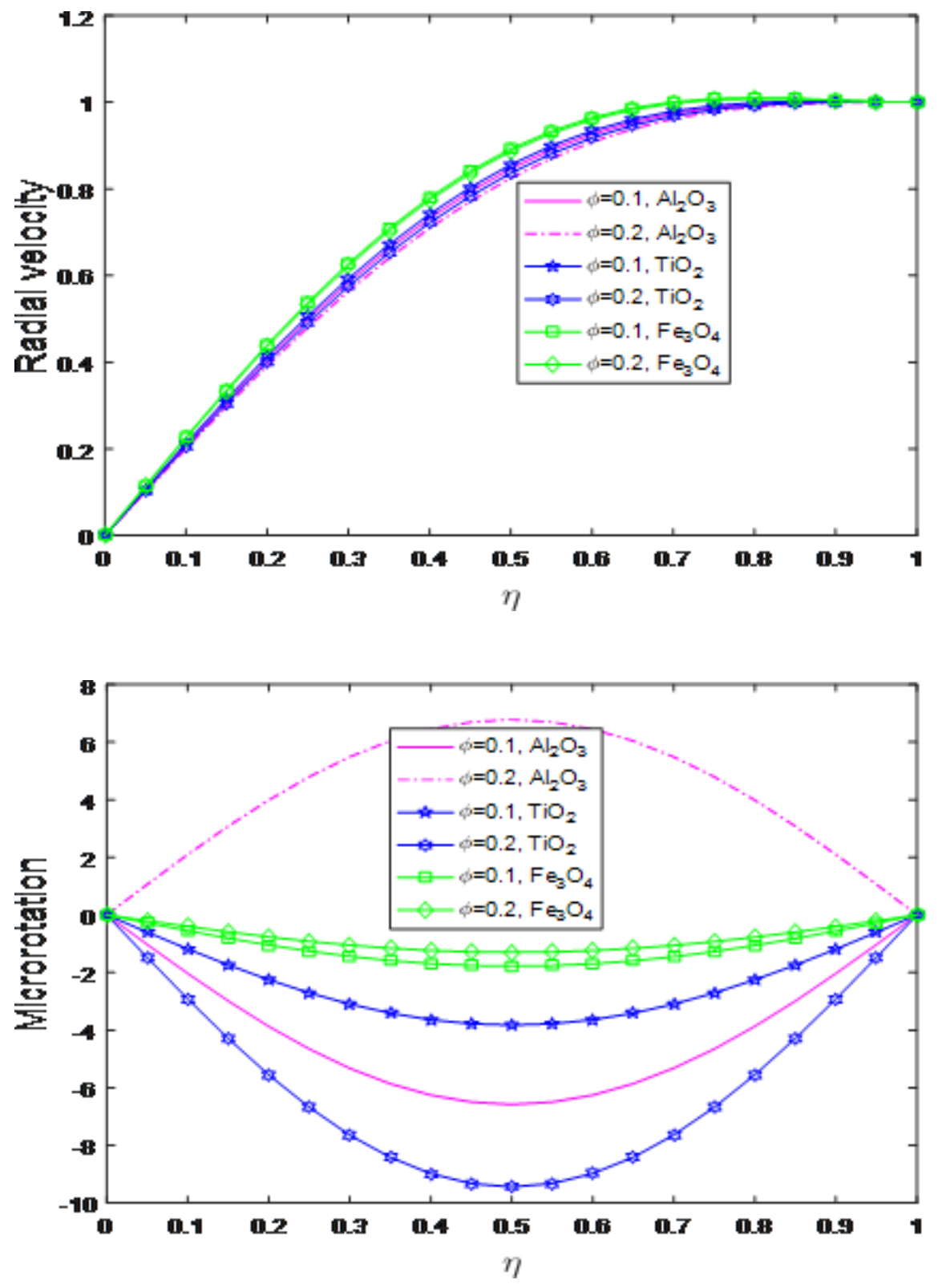


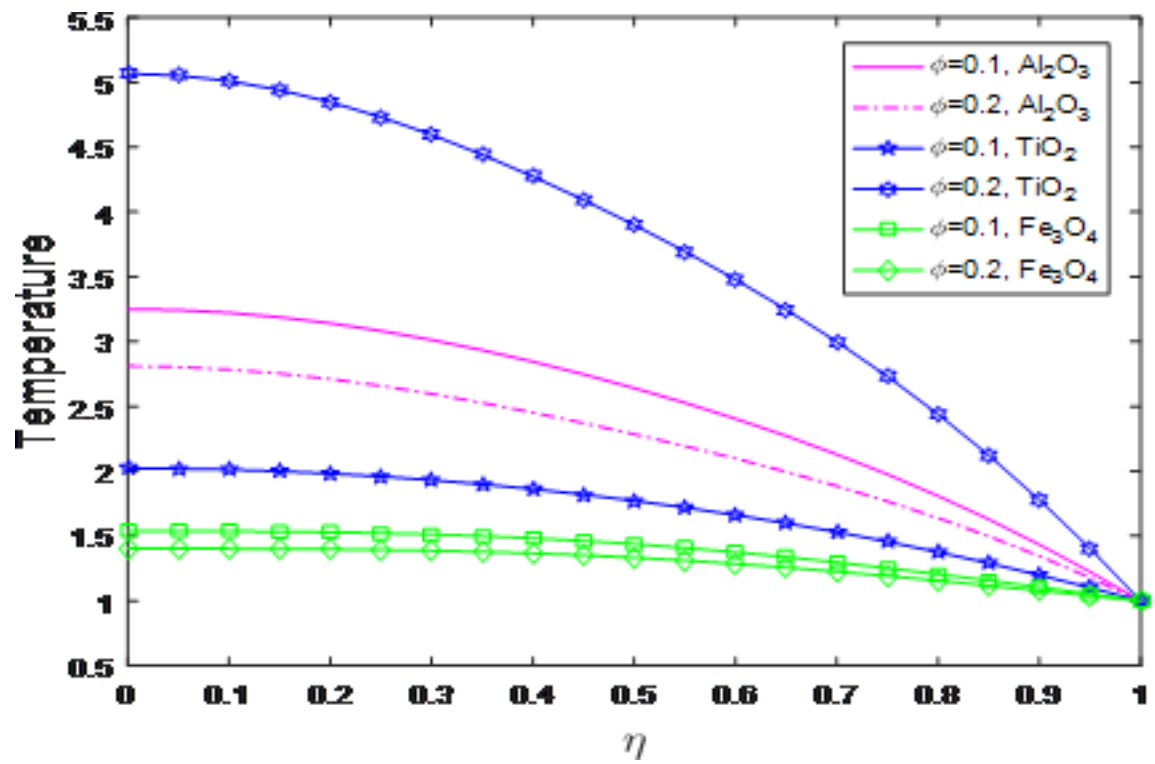

